\title{
Theory of interfaces and surfaces in wide-gap nitrides
}

\author{
Marco Buongiorno Nardelli, ${ }^{\text {a) }}$ Krzysztof Rapcewicz, and J. Bernholc \\ Department of Physics, North Carolina State University, Raleigh, North Carolina 27695-8202
}

(Received 29 March 1997; accepted 22 April 1997)

\begin{abstract}
A selection of the results of a theoretical investigation of the properties of interfaces and surfaces of the wide-gap III-V nitride semiconductors is reviewed. The electronic properties of wurtzite heteroepitaxial interfaces of AIN and GaN, incorporating the effects of strain, are discussed. In particular, we find that this interface is of type I and have calculated the valence-band offset to be $-0.57 \mathrm{eV}$. The surface energies and atomic geometries of the $2 \times 2$ reconstructions of the $(0001)$ face of $\mathrm{GaN}$ are also presented. In conditions which are rich in a given species, an adatom reconstruction of that species is found to be the most energetically favorable: for gallium-rich conditions, the reconstruction with a gallium adatom on a $T_{3}$ site is the most stable, while for nitrogen-rich conditions the reconstruction with a nitrogen adatom on the $\mathrm{H}_{3}$ site is energetically the most favorable. () 1997 American Vacuum Society. [S0734-211X(97)11904-7]
\end{abstract}

\section{INTRODUCTION}

Semiconductor heterojunctions find wide applications in microelectronics. These include lasers, photodetectors, highefficiency solar cells, and radiation-resistant integrated circuits. ${ }^{1}$ The III-V nitrides with their wide band gaps, high thermal stability and conductivity, and radiation resistance have long been viewed as attractive candidates for use in microelectronic devices. ${ }^{2}$

The recent demonstration of a GaN-based blue laser, which follows three decades of research, has served to highlight the potential of these materials. ${ }^{3}$ However, at present, the mechanisms underlying the workings of the blue laser are not understood. To this end and in order to develop other nitride-based devices, great effort is being expended to understand and control the materials properties of the wide gap nitrides.

\section{METHODOLOGY}

The standard ab initio plane-wave pseudopotential method $^{4-6}$ was used in the calculations of interface properties. To ensure convergence of the nitrogen pseudopotential, an energy cut-off for the plane-wave expansion of $50 \mathrm{Ry}$ was chosen. The equivalent of $10 k$-points for bulk and superlattice calculations in the zinc-blende structure ${ }^{7}$ and 6 $k$-points for calculations of the wurtzite structure ${ }^{8}$ were employed. A careful check of the convergence in both the size of the plane-wave basis and the number of special points was made. For the surface calculations, a multi-grid-based totalenergy method that uses a real-space grid as the basis was used. This approach has been used to study a wide range of systems involving large numbers of atoms. ${ }^{9}$ The ions were relaxed using the "fast-relax" algorithm. ${ }^{10}$

The Perdew-Zunger parametrization ${ }^{11}$ of the CeperleyAlder form ${ }^{12}$ of the exchange-correlation energy was chosen. Nonlocal, norm-conserving pseudopotentials ${ }^{13-15}$ were included using the Kleinman-Bylander approach. ${ }^{16}$ For gallium, a recently developed norm-conserving pseudopotential,

a)Electronic mail: nardelli@nemo.physics.ncsu.edu which includes a nonlocal core correction, ${ }^{17}$ and permits an efficient description without the need for an explicit treatment of the $d$ valence electrons was used. For nitrogen, a standard pseudopotential with a neutral configuration as the atomic reference for all states was employed. These pseudopotentials have been demonstrated to reproduce accurately the bulk properties of $\mathrm{GaN} .{ }^{18}$

The calculated bulk properties are presented in Table I. The theoretical lattice parameters of both the zinc-blende and wurtzite forms agree very well with the experiment; a similar level of accuracy is expected for the surface and interface calculations described below. The cohesive energy of GaN and $\alpha-\mathrm{Ga}$ is $10.42 \mathrm{eV}$ and $3.4 \mathrm{eV}$, respectively. The binding energy of nitrogen molecule is $5.87 \mathrm{eV}$ per nitrogen atom, in good agreement with other local density approximation (LDA) calculations. ${ }^{19}$ The theoretical heat of formation of $\mathrm{GaN}$ is thus $1.15 \mathrm{eV}$, in good agreement with the experimental value of $1.14 \mathrm{eV}$.

\section{INTERFACE PROPERTIES}

Interface behavior is very important in determining the properties of semiconductor devices. We have investigated the wurtzite (0001) GaN/AlN interface. Superlattices of AlN and $\mathrm{GaN}$ are expected to be strained because of the 3.5\% theoretical lattice mismatch between AlN and GaN (the experimental value is $2.7 \%$ ). The effects of the strain were incorporated using macroscopic elasticity theory. ${ }^{20}$ In this theory, each half of the heterojunction is treated as a strained bulk with a fixed in-plane lattice constant $a_{\|}$. The strain energy of the system is minimized, keeping the in-plane lattice constant fixed, to determine the perpendicular lattice constant, $c$, of the epilayer. For AlN and GaN, the calculated elastic constants used to determine the strains are given in Table II. Total energy calculations confirm the that macroscopic elasticity theory predicts well the perpendicular lattice constant for the epilayer. The residual relaxation of the atoms at the interface is negligible $(<0.05 \AA)$ and does not affect the band offset of the interface. ${ }^{18}$ 
TABLE I. Calculated bulk properties of zinc-blende and wurtzite nitride semiconductors. The values of the gap at the $\Gamma-\mathrm{pt}\left(E_{\Gamma}\right)$ and of the valenceband width $\left(\Delta E_{v b w}\right)$ are the LDA results. Note that the LDA indirect gap in zinc-blende AlN is $3.2 \mathrm{eV}$. Experimental values are in brackets and follow Ref. 35.

\begin{tabular}{cccc}
\hline \hline & AlN & GaN & InN \\
\hline \multicolumn{4}{c}{ zinc-blende } \\
$a_{0}(\AA)$ & $4.37(4.38)$ & $4.52(4.5)$ & $5.01(4.98)$ \\
$B_{0}$ (Mbar) & $2.02(2.02)$ & $1.70(1.90)$ & $1.58(1.37)$ \\
& \multicolumn{3}{c}{ wurtzite } \\
$a(\AA)$ & $3.09(3.11)$ & $3.20(3.19)$ & $3.55(3.54)$ \\
$c / a$ & $1.62(1.60)$ & $1.63(1.63)$ & $1.63(1.61)$ \\
$u$ (units of c) & $0.378(0.382)$ & $0.376(0.377)$ & 0.375 \\
$B_{0}$ (Mbar) & $1.99(2.02)$ & $1.69(1.95,2.37)$ & $1.62(1.26,1.39)$ \\
\hline \hline
\end{tabular}

The band offsets of the (0001) GaN/AlN strained heterojunction were studied following the procedure of Ref. 21. The valence band offset is divided into a band-structure contribution, which is the difference between the energies of the valence-band and conduction-band edges when the average electrostatic potentials of the epilayers are aligned, and the difference in the value of the average electrostatic potential $(\Delta V)$ in the two epilayers of the heterostructure. Strain will affect the electronic properties of the interface through the variation of the average electrostatic potentials and through the variations of the band edges (deformation potentials). The calculated valence-band offset in the case of an AlN in-plane lattice constant (strained $\mathrm{GaN}$ ) is estimated to be $-0.57 \mathrm{eV}, 22$ a value smaller than the result for the strained nonpolar (001) GaN/AIN interface. The ratio of the conduction-band to valence-band offset is $65: 20$. These results agree very well with the experimental measurements of the (0001) wurtzite interface. ${ }^{23}$

As spin-orbit effects in both AlN and $\mathrm{GaN}$ have been shown to be of the order of $20 \mathrm{meV}$, their difference, which enters into the calculations of the band offsets, is much smaller than other possible sources of systematic error. ${ }^{24}$ These include the neglect of the anion $p$ - and cation $d$-state repulsion ${ }^{25}$ and the well-known neglect of manybody effects in the LDA. In GaN/AlN interfaces, the inclusion of the $3 d$ electrons as valence electrons results in a constant shift of $0.2 \mathrm{eV}$, which is less than the experimental error, ${ }^{23}$ and does not change the character of the interface. Incorporating this shift gives results in agreement with previous work using a $d$ valence pseudopotential ${ }^{30}$ and an allelectron calculation. ${ }^{26}$ The importance of many-body effects on the band offsets is not known and awaits a future GW calculation.

TABLE II. Elastic constants calculated for zinc-blende AIN, GaN, and InN (in units of Mbar). The experimental values in brackets are from Ref. 36.

\begin{tabular}{cccc}
\hline \hline & AlN & GaN & InN \\
\hline$c_{11}$ & 3.01 & $2.61(2.64)$ & 2.14 \\
$c_{12}$ & 1.62 & $1.27(1.53)$ & 1.37 \\
\hline \hline
\end{tabular}

The wurtzite system displays pyroelectric and piezoelectric behavior. ${ }^{27}$ These effects will manifest themselves macroscopically in multiple quantum wells along those directions that do not have a perpendicular mirror plane. ${ }^{28,29} \mathrm{In}$ (0001) strained GaN/AlN, we observe a substantial electric field as has been previously noted by Satta and co-workers. ${ }^{30}$ We have calculated the spontaneous bulk polarization of unstrained AlN and the strain-induced polarization for the GaN epilayer, in order to distinguish the bulk pyroelectric and piezoelectric contributions to this field from that induced by the interface. A superlattice consisting of eight layers of wurtzite and six layers of lattice-matched zinc-blende was used. ${ }^{31}$ In unstrained zinc-blende, the bulk spontaneous polarization is rigorously zero. Further, zinc-blende-wurtzite interface does not introduce any chemical or geometrical perturbations. This construction, therefore, permits the unambiguous determination of the spontaneous polarization present in the wurtzite structure from the slope of the macroscopic average of the electrostatic potential. The spontaneous polarization $\left(P_{3}\right)$ of $\mathrm{AlN}$ and $\mathrm{GaN}$ in equilibrium is $-1.227 \mu \mathrm{C} / \mathrm{cm}^{2}$ and $-0.448 \mu \mathrm{C} / \mathrm{cm}^{2}$, respectively; the polarization of the strained $\mathrm{GaN}$ is $-0.454 \mu \mathrm{C} / \mathrm{cm}^{2}$. These values are comparable to the computed bulk polarization in $\mathrm{BeO} .{ }^{31}$ The effect of the interface dipole is small; the polarization in the GaN/AlN multiple quantum well is well described as the sum of the polarizations of the constituent epilayers of the multiple quantum well. The estimated contribution of the interface dipole (which includes the response of one epilayer to the field of the other) is $0.057 \mu \mathrm{C} / \mathrm{cm}^{2}$, which is of opposite sign and an order of magnitude smaller than the bulk polarizations. The computed value of the polarization in the superlattice agrees with that estimated from experiment by Martin et $a l^{23}$

\section{SURFACE RECONSTRUCTIONS}

The (0001) surface is a polar surface. The ideal surface has dangling bonds with charge distributions that are energetically unfavorable. If kinetically permitted, the surface atoms will relax and redistribute the "dangling-bond" charge density so as to satisfy the valences of all of the surface species. This can be achieved via structural relaxation (with a concomitant charge transfer between surface atoms). Recent experimental studies have revealed the presence of $2 \times 2$ reconstructions during growth. ${ }^{32}$ In this article, we restrict our attention to these reconstructions on the gallium-terminated face.

We considered eight different $2 \times 2$ reconstructions, namely the ideal relaxed structure and gallium vacancy, nitrogen adatom, gallium adatom, and nitrogen trimer, each on the $\mathrm{T}_{3}$ and $\mathrm{H}_{3}$ (hollow) sites (see Figs. 1 and 2). We find that adatom reconstructions are the most energetically favorable of the studied reconstructions. In particular, in the galliumrich case that the gallium adatom on the $T_{3}$ site has the lowest energy, while in the nitrogen-rich case the nitrogen adatom on the $\mathrm{H}_{3}$ is the most stable.

The slabs contained four bilayers of $\mathrm{GaN}$, three bilayers of which were relaxed. Relaxations in the third bilayer were 


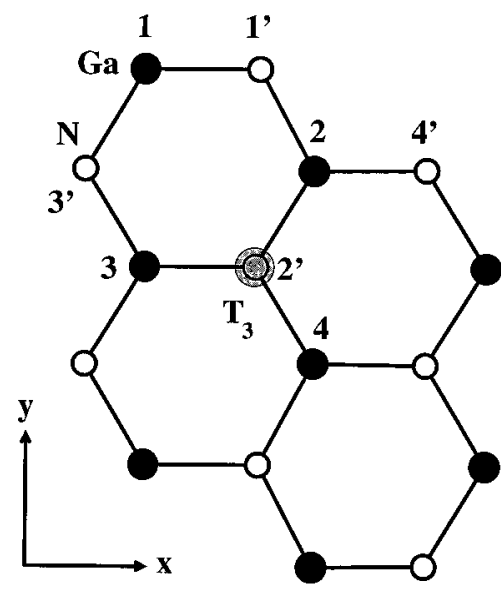

FIG. 1. Schematic top view of the $2 \times 2$ gallium-adatom reconstruction on the (0001) surface of GaN. The gallium adatom (in grey) sits above a nitrogen in the second layer on the $\mathrm{T}_{3}$ site. The numbers denote gallium atoms on the surface, while the primed numbers denote nitrogen atoms in the first subsurface layer.

negligible, indicating that a sufficiently thick slab was employed. Pseudo-hydrogens with $Z=0.75$ passivated the nitrogen-terminated face. ${ }^{33}$ If the work functions of the two surfaces are different, then the periodic boundary conditions of the supercell enforce a common electrostatic potential in the vacuum and result in an unphysical change in the electric potential in the vacuum region equal to the difference between the two work functions. A large vacuum region of 10 $\AA$ served to reduce the size of this field. Previous calculations that explicitly included a correction for this field did not show significant differences in the final equilibrium geometries,${ }^{34}$ and consequently field corrections were not included in our calculations. An orthorhombic supercell with grid spacing of $0.21,0.22$, and 0.20 a.u. in the $x, y$, and $z$ directions, respectively, was employed. The eight reconstructions mentioned above were studied using $\Gamma$-point sampling. For the lowest energy structures of each class, i.e., nitrogen

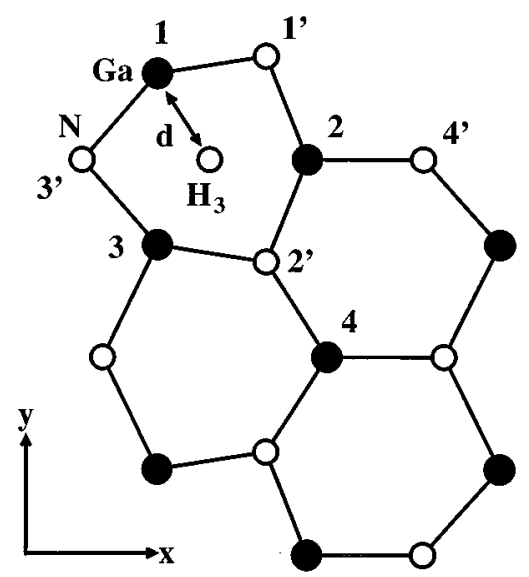

FIG. 2. Schematic top view of the $2 \times 2$ nitrogen-adatom reconstruction on the (0001) surface of GaN. The nitrogen adatom sits above the hollow $\left(\mathrm{H}_{3}\right)$ site. The numbers denote gallium atoms on the surface, while the primed numbers denote nitrogen atoms in the first subsurface layer.

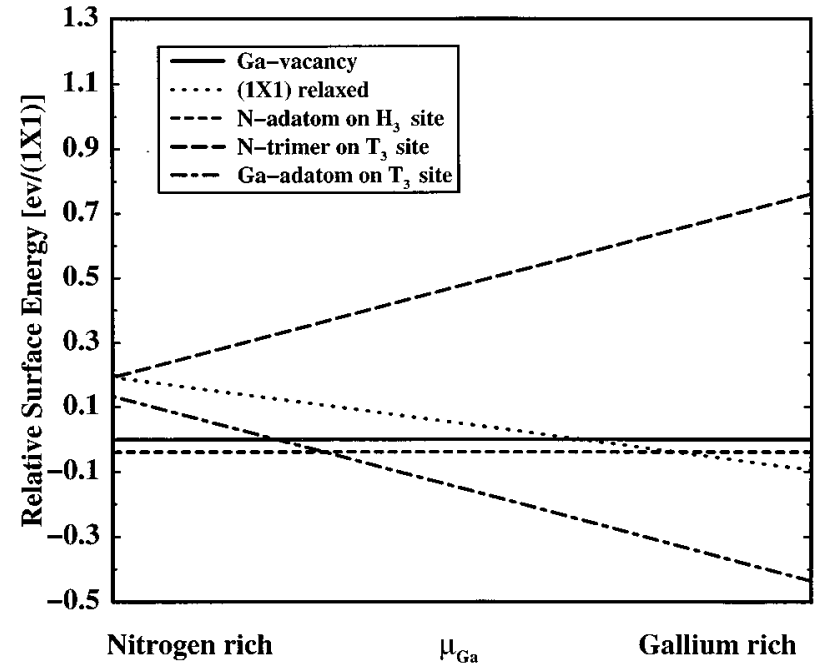

FIG. 3. Formation energy vs gallium chemical potential for the (0001) surface. The maximum chemical potential for $\mathrm{N}(\mathrm{Ga})$ is equal to the energy per atom calculated for $\mathrm{N}_{2}$ (bulk Ga). Two k-points are sampled in the irreducible Brillioun zone.

adatom on $\mathrm{H}_{3}$ site, nitrogen trimer on the $\mathrm{T}_{3}$ site, etc., a further calculation using two $k$-points in the irreducible Brillioun zone was carried out.

Figure 3 displays the relative surface energies for the five different reconstructions calculated using $2 \mathrm{k}$-points in the irreducible Brillioun zone. Under gallium-rich conditions and for most of the range of the chemical potential, the lowest energy reconstruction is the gallium adatom on the $T_{3}$ site, while under nitrogen-rich conditions, the reconstruction with the nitrogen adatom on the $\mathrm{H}_{3}$ site is preferred energetically.

The gallium-adatom reconstruction is shown in Fig. 1. The adatom sits on the $T_{3}$ site above the subsurface nitrogen atom; the gallium-nitrogen distance is $2.46 \AA$. The galliumadatom surface-gallium bond length is $2.40 \AA$, which is very close to the $\mathrm{Ga}$ dimer distance in $\alpha$-gallium (2.44 $\AA$ ). The bond angle between two gallium surface atoms and the adatom is $82^{\circ}$. The in-plane relaxation of the surface atoms is negligible; the only observed relaxation of the first bilayer is along the $z$ direction. The three gallium atoms bonded to the adatom remain coplanar to a significant degree, while the remaining unbonded gallium surface atom relaxes into the slab. The proximity of the gallium adatom to the nitrogen subsurface atom lowers the electrostatic energy and makes this particular configuration energetically more favorable than the gallium adatom reconstruction with the adatom on the hollow site.

The nitrogen-adatom reconstruction is shown in Fig. 2. The adatom sits on the hollow $\left(\mathrm{H}_{3}\right)$ site. The nitrogenadatom surface-gallium bond length is $2.0 \AA$ and is very close to the $\mathrm{Ga}-\mathrm{N}$ bond length distance in $\mathrm{GaN}$ bulk (1.96 $\AA$ ). The bond angle between two gallium surface atoms and the nitrogen adatom is $89^{\circ}$. The relaxation perpendicular to the surface is very similar to that in the gallium-adatom reconstruction. This configuration is stabilized because it separates the nitrogen adatom from the subsurface nitrogen atom and thereby lowers the electrostatic repulsion. 


\section{SUMMARY}

We have reviewed a selection of theoretical results for the properties of interfaces and surfaces of the gallium and aluminium nitrides. The electronic properties of wurtzite heteroepitaxial interfaces of AlN and GaN incorporating the effects of strain were discussed. The strained AlN/GaN interface is of type I and has a valence-band offset of -0.57 $\mathrm{eV}$. As a result of the pyro- and piezoelectric nature of the wurtzite nitrides, macroscopic polarization of $0.057 \mu \mathrm{C} /$ $\mathrm{cm}^{2}$, is present in multiple quantum wells along the (0001) direction. The surface energies and atomic geometries of the $2 \times 2$ reconstructions of the (0001) face of $\mathrm{GaN}$ were also investigated. Adatom reconstructions were found to be the most energetically favorable over the whole range of gallium chemical potential: under gallium-rich conditions, the reconstruction with a gallium adatom on a $\mathrm{T}_{3}$ site is the most energetically favorable, while under nitrogen-rich conditions the reconstruction with a nitrogen adatom on the $\mathrm{H}_{3}$ site has the lowest energy.

${ }^{1}$ R. Bauer and G. Margaritondo, Phys. Today 40, 27 (1987).

${ }^{2}$ M.-E. Lin, B. N. Sverdlov, S. Strite, H. Morkoç, and A. E. Drakin, Electron. Lett. 29, 1759 (1993).

${ }^{3}$ S. Nakamura, M. Senoh, S. Nagahama, N. Iwasa, T. Yamada, T. Matsushita, H. Kiyoku, and Y. Sugimoto, Jpn. J. Appl. Phys. 1 35, L74 (1996).

${ }^{4}$ M. Payne, M. Teter, D. Allan, T. Arias, and J. Joannopoulos, Rev. Mod. Phys. 64, 1045 (1992).

${ }^{5}$ W. Pickett, Comput. Phys. Rep. 9, 115 (1989).

${ }^{6}$ D. Remler and P. Madden, Mol. Phys. 70, 921 (1990).

${ }^{7}$ S. Froyen, Phys. Rev. B 39, 3168 (1989).

${ }^{8}$ D. J. Chadi and M. L. Cohen, Phys. Rev. B 8, 5747 (1973).

${ }^{9}$ E. L. Briggs, D. J. Sullivan, and J. Bernholc, Phys. Rev. B 52, R5471 (1995); E. L. Briggs, D. L. Sullivan, and J. Bernholc, ibid. 54, 14362 (1996).
${ }^{10}$ C. Wang, Q.-M. Zhang, and J. Bernholc, Phys. Rev. Lett. 69, 3789 (1992).

${ }^{11}$ J. Perdew and A. Zunger, Phys. Rev. B 23, 5048 (1981).

${ }^{12}$ D. Ceperley and B. Alder, Phys. Rev. Lett. 45, 566 (1980).

${ }^{13}$ G. Bachelet, D. Hamann, and M. Schlüter, Phys. Rev. B 26, 4199 (1982).

${ }^{14}$ D. Hamann, M. Schlülter, and C. Chiang, Phys. Rev. Lett. 43, 1494 (1979).

${ }^{15}$ D. Hamann, Phys. Rev. Lett. 40, 2980 (1989).

${ }^{16}$ L. Kleinman and D. Bylander, Phys. Rev. Lett. 48, 1425 (1982).

${ }^{17}$ S. Louie, S. Froyen, and M. L. Cohen, Phys. Rev. B 26, 1738 (1982).

${ }^{18}$ M. Buongiorno Nardelli, K. Rapcewicz, and J. Bernholc, Phys. Rev. B 55, R7323 (1997).

${ }^{19}$ B. G. Johnson, P. Gill, and J. A. Pople, J. Chem. Phys. 98, 5613 (1993).

${ }^{20} \mathrm{C}$. Van de Walle and R. M. Martin, Phys. Rev. B 34, 5621 (1986).

${ }^{21}$ A. Baldereschi, S. Baroni, and R. Resta, Phys. Rev. Lett. 61, 734 (1988).

${ }^{22}$ The band-offset was calculated following A. Munoz, N. Chetty, and R. M. Martin, Phys. Rev. B 41, 2976 (1990). The position of the interface was unambiguously determined from the position of the interface dipole.

${ }^{23}$ G. Martin, A. Botchkarev, A. Rockett, and H. Morkoç, Appl. Phys. Lett. 65, 610 (1994); 68, 2541 (1996).

${ }^{24}$ M. Suzuki, T. Uenoyama, and A. Yanase, Phys. Rev. B 52, 8132 (1995).

${ }^{25}$ S. Wei and A. Zunger, Phys. Rev. Lett. 59, 144 (1987).

${ }^{26}$ E. Albanesi, W. Lambrecht, and B. Segall, J. Vac. Sci. Technol. B 12, 2470 (1994)

${ }^{27}$ N. W. Ashcroft and N. D. Mermin, Solid State Physics (Saunder College, Philadelphia, PA, 1976), Ch. 27.

${ }^{28}$ D. Smith, Solid State Commun. 57, 919 (1986).

${ }^{29}$ A. Bykhovski, B. Gelmont, and M. Shur, Appl. Phys. Lett. 63, 2243 (1993); J. Appl. Phys. 74, 6734 ()1993.

${ }^{30}$ A. Satta, V. Fiorentini, A. Bosin, F. Meloni, and D. Vanderbilt, Mater. Res. Soc. Symp. Proc. 395, 515 (1996).

${ }^{31}$ M. Posternak, A. Baldereschi, A. Catellani, and R. Resta, Phys. Rev. Lett. 64, 1777 (1990)

${ }^{32}$ M. A. L. Johnson, Z. Yu, C. Boney, W. H. Rowland, Jr., W. C. Hughes, J. W. Cook, Jr., J. F. Schetzina, N. A. El-Masry, M. T. Leonard, H. S. Kong, and J. A. Edmond, Mater. Res. Soc. Symp. Proc. 449, 271 (1997).

${ }^{33}$ K. Shiraishi, J. Phys. Soc. Jpn. 59, 3455 (1990).

${ }^{34}$ J. Neugebauer and M. Scheffler, Phys. Rev. B 46, 16067 (1992).

${ }^{35}$ A. Wright and J. Nelson, Phys. Rev. B 50, 2159 (1994); 51, 7866 (1995).

${ }^{36}$ K. Kim, W. Lambrecht, and B. Segall, Phys. Rev. B 50, 1502 (1994). 